Fonctionnalité et symbolisation de l'arbre dans les contes ouest africains d'expression française

\title{
FONCTIONNALITE ET SYMBOLISATION DE L'ARBRE DANS LES CONTES OUEST AFRICAINS D'EXPRESSION FRANÇAISE
}

\section{Yao Lambert KONAN}

Résumé (Fr) : La représentation de l'arbre dans les textes oraux narratifs (mythes, légendes et contes) est liée étroitement au contexte ethnographique (milieu naturel, croyances, traditions et institutions). Cette étude a ainsi montré et confirmé que l'arbre est protecteur, nourricier et sacré. Au-delà de ces fonctions, ce végétal se trouve à la frontière des mondes, dans une sorte de "no man's land" entre l'espace des hommes et "l'autre monde", celui des forces surnaturelles ou encore celui des Esprits. Symbole de la culture et de la nature, symbole par excellence du passage du simple au complexe, de l'unique à la multiplicité, l'arbre se révèle initiatique car représentant pour le néophyte la mort et la résurrection. Ainsi, est-il lié à l'aventure humaine.

Mots-clés (Fr) : Africain; arbre; fonctionnalité; symbolisation; initiation.

\begin{abstract}
En): The representation of tree in the narrative oral texts (myths, legends and tales) is closely linked to ethnographic context (natural environment, believes, traditions and institutions). This study has thus showed and confirmed that tree is protective, nourishing and sacred. Beyond these functions, this plant is located to the frontier of worlds, in a kind of "No man's land" between the domain of humans and "the other world" those of supernatural forces or spirits. Symbol of culture and of the nature, symbol par excellence of the passage from simple to complex, from unique to multiplicity, the tree reveals to have an initiating power, because it represents for the neophyte the death and the resurrection. Thus, it is linked to human adventure.
\end{abstract}

Keywords (En): African; tree; functionality; symbolization; initiation.

Resumen (Es): La representación del árbol en los textos orales narrativos (mitos, leyendas, cuentos) está estrechamente ligada al contexto etnográfico (medio natural, creencias, tradiciones e instituciones). Así, este estudio ha mostrado y confirmado que el árbol es un protector, una nodriza y al mismo tiempo algo sagrado. Más allá de estas funciones, este vegetal está en la frontera de dos mundos, en una especie de "no man's land" entre el espacio de los hombres y "el otro mundo" que es el de las fuerzas naturales o, incluso, el de los espíritus. Simbolizando la cultura, la naturaleza y especialmente el paso de lo simple a lo complejo, de lo único a lo múltiple. El árbol se revela como iniciático porque representa la muerte y la resurrección del neófito, ligándose así, a la aventura humana.

Palabras claves (Es): africano; árbol; funcionalidad; simbolización; iniciación.

\section{INTRODUCTION}

L'Africain ${ }^{1}$, ontologiquement animiste, donne vie à tout ce qui l'entoure ${ }^{2}$. Vivant en symbiose avec tous les règnes (animal, végétal et minéral), il n'ignore pas la vie et la mort. Ainsi, le miraculeux, l'insolite, le terrifiant, parfois même le comique, font partie intégrante de son univers pour nourrir ce que l'on a appelé la littérature traditionnelle.

Cette littérature orale, par le biais de ses formes expressives narratives, met admirablement en scène des personnages humains, végétaux, animaux, allégoriques, supranaturels pour donner vie à ses récits. Mais tous ces êtres

\footnotetext{
${ }^{1}$ Celui de l'Afrique noire.

${ }^{2}$ Pour la tradition africaine, tout a une âme. Il y a une âme du minéral, une âme du végétal et une âme de l'animal. C'est ce qu'on appelle «les trois âmes », chaque règne ayant une âme unique. Cf. Amadou Hampâté Bâ, Njeddo Dewal Mère de la calamité, Abidjan, NEI, 1994, pp. 199-200.
} 
n'interviennent pas dans les mêmes proportions. Le végétal pourrait retenir l'attention de ce point de vue. Sa représentativité en tant qu'actant principal n'est pas toujours récurrente dans les textes oraux à l'instar des humains, des animaux et, quelquefois, des êtres anthropomorphes.

La présente réflexion s'intéresse à l'arbre ${ }^{3}$, saisit ce végétal à l'échelle d'adjuvant et se propose de dégager sa fonctionnalité et sa symbolisation dans la littérature des contes de l'espace ouest africain francophone.

\section{LES FONCTIONS DE L'ARBRE}

La quasi-totalité des textes explorés ${ }^{4}$ a permis un recensement des différents arbres rencontrés dans l'univers des récits. Les végétaux aux fréquentes occurrences sont le fromager, le baobab, le caïlcédrat et le jujubier. Certes, une pléthore de végétaux est aussi mentionnée. Cependant, la présence récurrente des quatre arbres s'explique par leur fonction sacralisante qui dépasse de loin les autres végétaux. Dans les contes, les fonctions de l'arbre sont diverses. L'arbre est protecteur, nourricier et sacré. Amadou Hampâté Bâ, dans ses textes Njeddo Dewal Mère de la calamité et Kä̈dara, met en exergue ces fonctions :

«Les trois grands de la brousse : un baobab, un caïlcédrat et un fromager. Leurs dômes étaient si épais et leurs branches si entremêlées qu'ils formaient une épaisse voûte de verdure sous laquelle régnait une ombre bienfaisante. Ils (les sept frères) se hâtèrent vers cet abri providentiel. (...) au pied du baobab se trouvait un grand canari rempli d'eau fraîche; entre les épaisses racines du fromager une marmite reposait sur trois pierres et sous le caïlcédrat plusieurs paniers contenant de la viande fraîche $\aleph^{5}$.

Le fromager a servi de refuge au "petit vieillard à la colonne déformée" qui n'est autre que l'une des incarnations de Kaïdara lui-même, dieu de l'or et de la connaissance. La sacralité de l'arbre correspond à ce que Pelissier appelle « enseigne» ou «identité » ${ }^{6}$. Pour Gastellu, cette fonction repose sur «le rôle religieux $\gg^{7}$. Dans la culture populaire (contes, légendes, récits, proverbes ...) l'arbre occupe une place importante, et se tissent autour de lui diverses représentations, croyances et images (l'arbre protecteur, fétiche, sauveur, ancêtre, identité, généalogie ...). Ce végétal est, de tous les thèmes symboliques, l'un des plus riches, des plus anciens, et des plus utilisés. Il est, en effet, l'axe du monde,

\footnotetext{
${ }^{3}$ Que Maillet et Bourgery définissent comme un végétal ligneux dont le développement adulte, en condition optimale, dépasse 7 mètres de haut. Cf. : L'arboriculture urbaine, Limoges, les presses du centre d'impression, 1993, p. 50.

${ }^{4}$ Kaïdara et Njeddo dewal Mère de la calamité de Amadou Hampâté Bâ ; Le Pagne noir de Bernard Binlin Dadié; Les Aventures de Tôpé l'Araignée de Touré Théophile Minan; La Belle histoire de Leuk-le-lièvre de Abdoulaye Sadji et Léopold Sédar Senghor; Les nouveaux contes d'Amadou Koumba de Birago Diop.

${ }^{5}$ Njeddo Dewal Mère de la calamité, Abidjan, NEI, 1994, p. 116.

${ }^{6}$ L'homme y lit son passé, son présent et son avenir à partir de l'alphabet que le novice apprend pendant son initiation et tout le long de sa vie. Il y apprend notamment à décrypter les messages qui fondent l'existence de sa nature et qui fondent sa propre existence, par exemple tel arbre est celui de l'invisibilité ; tel autre celui qui permet de vaincre le mal...cf « $\mathrm{L}$ ' arbre en Afrique, la fonction et le signe » in Cahier ORSTOM, Service Sciences Humaines, Volume XVII, N³-4, 1980, pp. 127-130.

${ }^{7}$ Gastellu J.M, «L'arbre ne cache pas la forêt ou usus, fructus et abusus », in Cahier ORSTOM, volume XVII, $\mathrm{N}^{\circ} 3-4,1980$, pp. 279-282.
} 
autour de lui s'organise le Cosmos Vivant. Elévation de l'âme, ascension initiatique, l'arbre est présent dans les genèses même de toute religion. Il unit les univers chtonien et ouranien, et symbolise le lieu de pacification ${ }^{8}$.

Il s'agit donc du rôle psychologique et social, que joue l'arbre en tant que facteur de cohésion sociale. En Afrique, comme d'ailleurs en Inde ${ }^{9}$ ou d'autres pays ${ }^{10}$, certains arbres revêtent un caractère imaginaire et social exacerbé, l'on parle alors de « sacré ».

L'arbre, en effet, est dit sacré lorsqu'il s'agit du fromager ou du baobab, car ces végétaux sont, selon Claude Olivier Djongang, consacrés par un aïeul. Ils constituent par excellence les lieux des rites sacrificiels : «Ces arbres abritent les crânes des ancêtres protecteurs, et c'est là que sont déposées les offrandes (sel, huile de palme, aliments cuits, animaux à sacrifier...) » ${ }^{11}$. Le héros d'Amadou Hampâté Bâ, Bâgoumâwell, confronté à une difficulté, recourt au jujubier ancestral :

«Pour recevoir une révélation, Bâgoumâwell se rendit sous le jujubier sacré de Heli et Yoyo. (...) Il sortit d'un sac, le crâne parleur dont il avait hérité de son grand-père Bâwâm'ndé $»^{12}$.

L'arbre sacré se révèle donc comme la demeure des esprits ancestraux.

Le baobab et le fromager sont les endroits de prédilection des génies malveillants ou bienveillants: "Tôpé demandait à sa femme d'aller en consultation chez le grand devin dont les génies inspirateurs habitaient le grand baobab $»^{13}$. Ces êtres extraordinaires, surtout ceux animés de bons sentiments, viennent en aide aux humains lors des difficultés, notamment au moment des disettes. Les contes de la faim ont en effet, très souvent, montré que la nourriture qui fait défaut aux personnages au village est trouvée en brousse au pied des "géants de la brousse".

Ces végétaux assurent la survie du personnage principal en lui favorisant l'accès à l'autre monde. L'arbre, dans ces récits de la faim, est le lieu de rencontre entre le héros et les êtres supranaturels.

\footnotetext{
${ }^{8}$ Cf. : L'usage fréquent de l'arbre de village (baobab, jujubier ...) comme lieu de l'effervescence sociale, la place communautaire, celle des réunions publiques et des sacrifices.

${ }^{9}$ Le figuier cosmique, Asvattha (ou arbre Pipal, ficus religiosa) est le représentant sur terre de Brihaspati, qui n'est autre que Jupiter. C'est un arbre hautement sacré, car il est dédié à la Trimurti (trois dieux) : Brahmâ, Shiva et Vishnu. Il abrite l'âme des défunts, et on lui rend un culte de fécondité. Cf. : Jean Chevalier et Alain Gheerbrant, Dictionnaire des Symboles. Mythes, rêves, coutumes, gestes, formes, figures, couleurs, nombres, Paris, Robert Laffont, coll. "Bouquins", t. I, chap. IX : "Le culte des arbres", p. 268-289, p. 268-289 (esprit des arbres), p. 289-296 (Pouvoirs bienfaisants des esprits des arbres).

${ }^{10}$ Mircea Eliade évoque un culte des arbres liés aux dieux Attis et Cybèle, en Grèce antique. Cf. : Histoire des croyances et des idées religieuses, tome II, Paris, Payot, 1978, p. 275.

${ }^{11}$ «Fonctions séculaires de l'arbre et dynamiques actuelles en Afrique subsaharienne : le cas du pays Bamiléké dans l'ouest camerounais » in Actes du séminaire «Etapes de recherche en paysage ", $\mathrm{n}^{\circ} 6$, Ecole nationale supérieure du paysage, Versailles 2004, p. 20.

${ }^{12}$ Njeddo Dewal Mère de la calamité, op. cit., p. 165.

${ }^{13}$ Les Aventures de Tôpé l'Araignée, op. cit., p. 75.
} 
Dans Le Pagne noir de Bernard Dadié, le héros, Kacou Ananzè ${ }^{14}$, est secouru par les animaux (le silure et l'écureuil) considérés comme des êtres providentiels. "Le miroir de la disette", s'ouvre sur un monde de désolation, de tristesse, un univers tragique : «La famine donc était au village (...) Chaque jour (elle) devenait plus atroce (...) La famine donnait la main à la mort (..) tant les êtres mouraient, mouraient $»^{15}$. Kacou Ananzè fut épargné momentanément de cette calamité par un silure qui lui recommanda de grimper à la douzième branche d'un fromager. Il fut ainsi projeté dans le pays de Cocagne.

Le même scénario se répéta, cette fois-ci, avec l'écureuil dans le «Araignée et la Tortue ». Ici, dans ce conte, aucun arbre n'est nommé spécifiquement, mais après plusieurs traversées d'espaces verdoyants, le héros arrive au pays de la bombance, le village de l'écureuil.

L'on peut retenir que l'arbre apparaît comme un adjuvant, puisqu'il relance les péripéties de l'intrigue en portant secours à l'actant principal. L'acte posé lui confère la fonction nourricière. Dans certains contes, il joue le rôle de justicier et sa tâche est liée à une action réparatrice et, comme tel, sert d'instrument de ruse au héros. "Le néré de Dissia"16 s'inscrit dans cette perspective. Refusant de partager les fruits de cet arbre avec les membres de sa communauté en raison de sa gourmandise légendaire, corollaire d'un égoïsme foncier, symptomatique d'une idéologie individualiste contraire aux valeurs communautaires, Dissia l'Hyène est châtié par Tôpé l'Araignée. Ce dernier, en effet, ligota l'égoïste à son arbre et distribua les grappes de gousses à tous les habitants du village.

En dépit de sa passivité apparente, l'arbre agit donc de façon sélective, en connaissance de cause, comme s'il était au-delà de l'enjeu contingent du conflit, un outil, voire le représentant de quelque justice immanente. Justicier ici passif mais partial, l'arbre met en valeur, par l'ambiguïté de son rôle, le message des récits concernant le rétablissement souhaité et inéluctable de l'ordre social perturbé par un méfait.

Si la fonction justicière passive de l'arbre apparaît dans les textes de Touré Théophile Minan, le contraire s'opère dans ceux de Birago Diop, notamment dans le conte "La cuiller sale". L'héroïne Binta, orpheline de mère, victime des exactions de sa marâtre, entre en conversation avec le jujubier, après s'être arrêtée au pied de cet arbre qui gaulait lui-même ses fruits :

« - où vas-tu donc si seule et si tard, mon enfant?

S'enquit le jujubier.

- Ma marâtre m'a envoyée laver cette kôk à la mer de Danyane,

expliqua la petite fille.

- Que le chemin de Dieu guide tes pas, souhaita l'arbre.

Et il prit une grosse poignée de jujubes qu'il offrit à l'orpheline. ${ }^{17}$

Par ailleurs, dans les récits oraux, ce végétal sert de limite, de frontière entre les mondes naturel et supranaturel : ce que la cosmogonie peule désigne par «pays

\footnotetext{
${ }^{14}$ Nom de l'araignée en Akan, précisément dans l'ethnie N'zima, peuple lagunaire de la Côte d'Ivoire situé dans le Sud Est et Tôpé, l'araignée, en langue Tagbanan, au Centre Ouest.

15 "Le miroir de la disette", p. 8-9, in Le Pagne noir, Paris, Présence Africaine, 1955.

${ }^{16}$ Cf. : Les Aventures de Tôpé l'Araignée, op.cit., p. 81.

17 "La Cuiller sale", p. 179, in Les Nouveaux contes d'Amadou Koumba, op.cit.
} 
de clarté où logent les vivants (et) le pays de pénombre où se meuvent les esprits, les génies et les autres forces surnaturelles ${ }^{18}$.

Si le personnage trouve assistance par l'apport des génies logés dans les contreforts des gros arbres et qui lui facilitent l'accès au pays des Esprits, il n'en demeure pas moins vrai qu'aux prodigalités des génies, il y a toujours un mais, une condition. Le héros, en effet, peut toucher à tout sauf à un objet, ou bien il a la permission de parler à tous sauf à un être. La reine du pays magique dit à Araignée : « Tu peux tout faire dans mon royaume, tout faire dans mes palais, mais ce que tu ne dois jamais faire, c'est te regarder dans le miroir qui est làbas $\gg{ }^{19}$. Au village de l'Ecureuil, la Tortue est plutôt interdite à Kacou Ananzè. Pourquoi ces interdictions ? Le second point de cette réflexion apportera des éclaircissements.

\section{LE SYMBOLISME DE L’ARBRE}

La pensée africaine n'est pas une philosophie au sens occidental, mais une manière de penser, une vision complète de l'individu au sein de la société et dans le monde. Cette pensée s'exprime dans la vie sociale, dans la vie religieuse, dans les différentes techniques par une série de symboles polyvalents dont le déchiffrement est du plus haut intérêt pour la connaissance de l'homme africain et de l'homme en général. Quel est le symbole qui se dégage de l'arbre ? Comment dans l'imaginaire ${ }^{20} 1$ 'Africain saisit-il ce végétal ?

De toute évidence, les récits analysés, au-delà de leur apparente hétérogénéité thématique, dégagent un symbolisme riche et complexe en raison du contexte culturel local. Ainsi, dans l'espace savanicole et même à la limite sahélienne où le royaume végétal est en grande partie dominé par la présence du baobab, ce végétal est le symbole maternel, car il assure la survie du héros ${ }^{21}$ en lui apportant de la nourriture et la protection.

La zone forestière retient, quant à elle, comme arbre magique, le fromager. $\mathrm{Ce}$ végétal est, en effet, l'arbre au trésor; c'est une sorte de sésame débouchant sur l'abondance. Il faut, toutefois, retenir que l'arbre symbolise l'autre monde; un monde avec ses beautés, ses richesses, mais un univers régi par ses lois où seul l'initié peut aller et venir sans crainte. L'arbre est loin d'être un simple élément décoratif dans le tissu narratif. Sa signification va au-delà de ses apparences et ce qui le particularise le plus, c'est son caractère ambivalent. Il entretient, en effet, une pluralité de facettes et d'attributs que l'on peut résumer à un ensemble de binômes: il est invariablement le lieu de coexistence entre le naturel et le surnaturel, entre le passé et le présent, entre le visible et l'invisible, entre les vivants et les morts. Ce végétal se trouve donc, souvent, au centre de la « dialectique de la vie et de la mort », car symbolisant l'initiation.

La conduite atypique de Kacou Ananzè le conduit à son expulsion de l'eldorado. En opposant son autorité individuelle à l'autorité collective, il trouble

\footnotetext{
${ }^{18}$ Cf. : Kaïdara, p. 8.

19 "Le miroir de la disette", op., cit, p. 15.

${ }^{20}$ L'imaginaire suppose la capacité d'utiliser le symbole pour exister. C'est le fait de voir dans les choses ce qu'elles ne sont pas, c'est-à-dire de les voir autrement.

21 "Leuk chez les aveugles", p. 54, in La Belle histoire de Leuk-le-lièvre, Dakar, NEA, 1990.
} 
par conséquent le réseau social et se signale comme un danger pour l'intégration communautaire. Ce heurt entre valeurs personnelles et valeurs sociales montre et démontre que l'individu a raté son initiation. Ce n'est pas le cas de Bâgoumâwell qui se réfère au jujubier ancestral. Son geste permet d'affirmer que cet arbre représente la sagesse et la coutume, données par les ancêtres.

Cet arbre ancestral ${ }^{22}$ lui a permis de résoudre l'énigme à laquelle il était confronté. Ce végétal met ainsi l'accent sur divers aspects d'une commune réalité - la réalité des ancêtres : le végétal est ontologiquement lié à l'homme ${ }^{23}$, justifié par la réflexion d'Hubert Deschamps : « la nature apparaît à l'Africain si distincte de lui-même qu'il attribue aux animaux, aux plantes, voire même aux minéraux et aux objets, ses propres qualités, ses besoins et ses désirs $»^{24}$. Cette importance de l'arbre justifie manifestement le fondement du totémisme végétal ${ }^{25}$ et révèle un pan de la profonde croyance traditionnelle africaine qui autorise et fonde l'animisme ${ }^{26}$. Il existe, en effet, un lien profond entre l'Africain traditionnel et l'arbre, ce que Julia Kristeva appelle «l'unité mythique » qui est le fondement de la vision du monde africaine. Au-delà du fait que ce végétal incarne l'archétype exemplaire de la végétation telle que trouvée dans l'Afrique forestière, il est aussi l'élément qui inspire respect et soumission, étant donné qu'il est rattaché à l'homme par un certain nombre de faisceaux mystiques. Cette fonction ésotérique se présente comme un nœud par lequel la force vitale de la communauté est renforcée. L'arbre acquiert ainsi une personnalité définie, il est le lieu d'intersection entre les ancêtres et le clan.

\section{CONCLUSION}

Cette étude a révélé un rapport de collusion entre l'arbre et l'homme fondé sur un mécanisme plurifonctionnel. Considéré comme l'espèce la plus aboutie de l'évolution végétale, à l'image de l'homme pour la créature animale, l'arbre est protecteur, nourricier et ancestral ${ }^{27}$. Les textes oraux ont saisi ce végétal comme,

\footnotetext{
${ }^{22}$ Le jujubier (Ziziphus mauritiana, Ziziphus Spina-cristi et Ziziphus lotus) est respecté pour avoir caché le prophète Mahomet à la vue de ses ennemis grâce à ses frondaisons. Il est aussi utilisé dans les tombes pour empêcher grâce à ses branches épineuses, les hyènes d'approcher les dépouilles. $\mathrm{Cf}$ : Claude Olivier Djongang dans «Fonctions séculaires de l'arbre et dynamiques actuelles en Afrique subsaharienne », op. cit., p. 20.

${ }^{23}$ Cf : Hirsch Charles et Davy Marie-Madeleine, L'Arbre, Paris, Philippe Lebaut, 1997. Ils affirment à ce sujet que : «La mort même ne brise pas les attaches et l'arbre donne une impulsion à la vie de l'au-delà. (...) C'est dès lors un arbre enraciné dans un passé encore là, culminant dans un futur déjà là, et dont le temps ordinaire, celui des horloges, mais calciné, putréfié, dissous, enfin distillé, devient la sève, le breuvage de l'authentique immortalité. », pp. 18 et 60 ; et Durand Gilbert : «Rien n'est donc plus fraternel et flatteur au destin spirituel ou temporel de l'homme que de se comparer à un arbre séculaire, contre lequel le temps n'a pas de prise, avec lequel le devenir est complice de la majesté des frondaisons et de la beauté des floraisons. ", dans Les Structures Anthropologiques de l'Imaginaire, Paris, Dunod, 1984 (10 ème édition), p. 396.

${ }^{24}$ Hubert Deschamps, Les Religions d'Afrique noire, Paris, PUF, 1965, p. 68.

${ }^{25}$ Sigmund Freud, Totem et Tabou, Paris, Payot, 1973.

${ }^{26} \mathrm{Au}$ sens étroit du mot, l'animisme est la théorie des représentations concernant l'âme ; au sens large, la théorie des êtres spirituels en général. C'est le fait pour l'homme de donner vie aux éléments qui l'entourent. Cf. : Totem et tabou de Sigmund Freud, p. 89.

${ }^{27}$ Jean Pliya, L'Arbre fétiche, Yaoundé, Clé, 1971, p. 13
} 
non seulement frontière de deux mondes, mais aussi et surtout, comme symbole de l'initiation permettant la socialisation de l'individu.

Intermédiaire entre la terre et les règnes supra et infra terrestres et entre la nature et la société, l'arbre dans les contes se présente, en effet, en qualité de sésame, adjuvant, demeure des esprits ancestraux et de prétexte pour stigmatiser les vices et défauts et célébrer les vertus.

\section{BIBLIOGRAPHIE}

Corpus

BA Amadou Hampâté, Kä̈dara, Abidjan, NEI, 1994.

BA Amadou Hampâté, Njeddo Dewal mère de la calamité, NEI, 1994.

DADIE Binlin Bernard, Le Pagne noir, Paris, Présence Africaine, 1955.

DioP Birago, Les Nouveaux contes d'Amadou Koumba, Paris, Présence Africaine, 1961.

MINAN Touré Théophile, Les Aventures de Tôpé l'Araignée, Abidjan-Dakar, CEDA, 1983.

SADJI Abdoulaye; SENGHOR Léopold Sédar, La Belle histoire de Leuk-le-lièvre, Dakar, Edicef-NEA, 1990.

Ouvrages de référence et études sur l'arbre

Chevalier Jean; GHeERBRANT Alain, Dictionnaire des symboles. Mythes, rêves, coutumes, gestes, formes, figures, couleurs, nombres, Paris, Robert Laffont, collection «Bouquins », tome I, 1982.

DESCHAMPS Hubert, Les Religions d'Afrique noire, Paris, PUF, 1965.

DJONGANG Claude Olivier, «Fonctions séculaires de l'arbre et dynamiques actuelles en Afrique subsaharienne: le cas du pays Bamiléké dans l'Ouest camerounais », in Actes du séminaire «Etapes de Recherche en paysage », Versailles, 2004.

DURAND Gilbert, Les Structures Anthropologiques de l'Imaginaire, Paris, Dunod, 1984, $10^{\text {ème }}$ Edition.

ELIADE Mircea, Histoire des croyances et des idées religieuses, tome II, Paris, Payot, 1978.

FREUD Sigmund, Totem et Tabou, Paris, Payot, 1973.

GASTELLU Jean-Marc, «L'arbre ne cache pas la forêt ou usus, fructus et abusus », in : Cahier ORSTOM, volume XVII, n³-4, 1980.

HIRSCH Charles ; DAVY Marie-Madeleine, L’Arbre, Paris, Philippe Lebaud, 1997.

KRISTEVA Julia, Le Texte du roman, Paris, Mouton, 1970.

MAILlET Laurent ; BOURGERY Corinne, L'Arboriculture urbaine, Limoges, Les presses du centre d'impression, 1993.

PELISSIER Paul, «L'arbre en Afrique, la fonction et le signe », in : Cahier ORSTOM, Services Sciences Humaines, volume XVII, n³-4, 1980.

PLIYA Jean, L’Arbre fétiche, Yaoundé, Clé, 1971. 